\title{
Analysis of a Multilevel Iterative Method for Nonlinear Finite Element Equations*
}

\author{
By Randolph E. Bank and Donald J. Rose
}

\begin{abstract}
The multilevel iterative technique is a powerful technique for solving the systems of equations associated with discretized partial differential equations. We describe how this technique can be combined with a globally convergent approximate Newton method to solve nonlinear partial differential equations. We show that asymptotically only one Newton iteration per level is required; thus the complexity for linear and nonlinear problems is essentially equal.
\end{abstract}

1. Introduction. In this discussion we present an extension of a multilevel iterative method for linear elliptic equations to nonlinear boundary value problems. In particular, we show how to use an approximate-Newton multilevel scheme to solve the discrete nonlinear systems of equations which arise from a standard weak formulation of the nonlinear partial differential equation.

The framework of our analysis combines the multilevel iterative methods for linear finite element equations discussed in Bank and Dupont [2] and Bank [3] with the global approximate Newton setting of Bank and Rose [4], [5]. Under appropriate conditions of elliptic regularity, we show that both the continuous and discrete solutions exist and that our scheme converges to an approximation within the discretization error of the continuous problem in time (and also space) proportional to the largest discrete problem. That is, we can compute in time $O\left(N_{j}\right)$ an approximation which is $O\left(N_{j}^{-q}\right)$ accurate, where $q$ is the appropriate exponent for the $N_{j}$-dimensional finite element spaces $\Re_{j}$.

In Section 2, we set up the weak (variational) form of the nonlinear boundary value problem. Using this formulation, we then specify, in Section 3, our regularity assumptions on the smoothness of the nonlinear operator. These assumptions are motivated by the generalized Lax-Milgram analysis presented by Babuška and Aziz in [1] and our previous analysis in [5]. Our main result here is that, asymptotically, we need compute only one approximate Newton iteration per level (refinement), provided that the approximate and exact Newton steps agree to some tolerance which is independent of the level. This implies that the total cost of solving a nonlinear problem of size $N_{j}$ is bounded by $C \cdot F\left(N_{j}\right)$, where $F\left(N_{j}\right)$ is the cost of solving a linear problem of size $N_{j}$ and $C \cong 1 . F\left(N_{j}\right)=O\left(N_{j}\right)$ for the linear multigrid methods described in [2], [3].

Received April 23, 1981; revised November 17, 1981 and January 29, 1982.

1980 Mathematics Subject Classification. Primary 65H10, 65F10, 65N20.

*This work was supported in part by the Office of Naval Research under grants N00014-80-C-0645, and N00014-76-C-0277. 
In Section 4, we consider the case where the linear approximate-Newton equations are solved by the $j$-level scheme of [2], [3], and we complete the analysis for the time bound cited above. We illustrate our analysis with an example boundary value problem of the form

$$
\begin{array}{ll}
L(u)=0 & \text { in } \Omega \subseteq R^{2} \\
\partial u / \partial n=0 & \text { on } \partial \Omega
\end{array}
$$

where

$$
L(u)=\nabla a \nabla u+f(x, u, \nabla u)
$$

A numerical example is given in Section 5.

Our approach for extending multilevel methodology to nonlinear operators using an approximate-Newton iterative scheme differs in several respects from other approaches recently reported or under investigation. We discuss briefly the relation of our scheme to those of Brandt and McCormick [8], Hackbusch [10], and Mansfield [12].

A common thread in our approach, and those of [8], [10], is the consideration of a sequence of discrete nonlinear problems, say, $L_{j}\left(u_{j}^{*}\right)=0$, where the $u_{j}^{*}$ are successively more accurate approximations of the solutions of the nonlinear operator $L(u)=0$. As a consequence, the representation of $u_{j}^{*}$ in the space containing $u_{j+1}^{*}$ is such that $L_{j+1}\left(u_{j}^{*}\right)$ is relatively small. This motivates the choice of taking $u_{j}^{s_{j}}$, for some iteration index $s_{j}$, as the initial guess in an iterative method to solve $L_{j+1}\left(u_{j+1}^{*}\right)$ $=0$. The integer $s_{j}$ is chosen such that the error $\left\|u_{j}^{*}-u_{j}^{s_{j}}\right\|$ is accurate to within the discretization error. Thus $L_{j+1}\left(u_{j}^{s_{j}}\right)$ will also be relatively small, and consequently the iterative method should require $s_{j} \leqslant s$ steps (independent of $j$ ) for each mesh level $j$.

Usually the iterative method selected to compute the $u_{j}^{k}, 1 \leqslant k \leqslant s_{j}$, is subtle and recursively winds its way through a sequence of coarser mesh levels; the details need not concern us here. However, each choice of such an iterative method leads to a different ' $j$-level' strategy. The $j$-level strategy can be based on a nonlinear iteration, such as the nonlinear Gauss-Seidel method advocated in [8], or on a nonlinear Picard type iteration used in [10]. These schemes make no use of Jacobian information.

In contrast, we use a $j$-level strategy based on a linear iteration after choosing a linear system to represent the Jacobian. Since asymptotically $s_{j}=1$ for this procedure, this strategy will usually require substantially fewer function evaluations of the $L_{j}$. On the other hand, for problems where the Jacobian is difficult to compute, our method becomes less attractive.

The recent paper by Mansfield [12] takes a different approach. In order to solve $L_{j}\left(u_{j}^{*}\right)=0$, for some fixed mesh index $j$, she considers a one parameter embedding $h_{j}(v, \lambda)=0,0 \leqslant \lambda \leqslant 1$, such that $h_{j}(0,0)=0$, and $h_{j}\left(u_{j}^{*}, 1\right)=L_{j}\left(u_{j}^{*}\right)=0$. The solution is continued from $v=0$ to $v=u_{j}^{*}$ by solving $h_{j}\left(v_{i}, \lambda_{i}\right)=0$, where $0=\lambda_{1}$ $<\lambda_{2}<\cdots<\lambda_{m}=1$. The $\lambda_{i}$ are chosen such that $v_{i}$ can be computed by Newton's method using $v_{i-1}$ as the initial iterate. Mansfield proves that the error $\left\|u_{j}^{*}-u\right\|$, where $L(u)=0$, is accurate to the discretization order, and the number of continuation steps, $m$, is independent of the mesh. Furthermore, by showing that the number of Newton steps, $s_{i}$, to obtain the computed $v_{i}$ satisfies $s_{i} \leqslant s$, independent of the 
mesh, and by using a linear $j$-level iterative scheme for the Newton equations, she obtains an $O\left(N_{j}\right)$ time bound. Assuming that these computed approximations to the $u_{j}^{*}$ are accurate to the discretization error, this result is analogous to our theorem in Section 4. Note that this method may require $m \cdot s$ linear systems be solved on the finest mesh. Our results would suggest an alternative in which one continues from $\lambda=0$ to $\lambda=1$ on the coarsest mesh only, thereby obtaining $u_{1}^{s_{1}}$. One then refines the mesh for $\lambda=1$ and obtains the sequence $u_{j}^{s_{j}}$ on the finer meshes. This would asymptotically require only one linear system be solved on the finest mesh.

Multilevel iteration is a general, powerful technique for solving nonlinear operator equations which can be approximated by an orderly sequence of discrete nonlinear systems. The linear multigrid schemes of Brandt [7], Hackbusch [9], Nicolaides [13], and possibly others, could be adapted in a similar manner to the one proposed here and would yield methods with similar properties. We have found our particular procedure to be effective on a variety of nonlinear PDE's; the implementation was a reasonably straightforward extension of the one described in [6] for linear problems.

2. Preliminaries. To introduce ideas, we consider a weak form of the example nonlinear elliptic boundary value problem (1.1)-(1.2): find $u \in H^{1}(\Omega)$ such that

$$
\begin{aligned}
& a(u, v)=0 \text { for all } v \in H^{1}(\Omega), \\
& a(u, v)=\int_{\Omega} a \nabla u \cdot \nabla v+f(x, u, \nabla u) v d x .
\end{aligned}
$$

Here $H^{1}(\Omega)$ denotes the usual Sobolev space equipped with the norm

$$
\|u\|_{1}^{2}=(u, u)_{1}, \quad(u, v)_{1}=\int_{\Omega} \nabla u \nabla v+u v d x .
$$

We will defer our discussion of nonlinear elliptic problems such as (2.1) until Section 4. In this section and the next, we prefer to deal with a more abstract problem for which (2.1) is a special case.

Let $g$ be a mapping of a Hilbert space $H$ onto itself. Equip $H$ with an inner product $(u, v)$ and norm $\|u\|^{2}=(u, u)$. We consider the following problem: find $u^{*} \in H$ such that

$$
\left(g\left(u^{*}\right), v\right)=0 \text { for all } v \in H .
$$

In the example above, $g$ is defined implicitly via the Riesz representation theorem, $H=H^{1}(\Omega)$, and the norm and inner product are given by (2.2).

We shall (formally) apply an approximate Newton method to (2.3). Starting from some initial guess $u^{0} \in H$, we compute a sequence of iterates $u^{k} \in H, k=1,2,3, \ldots$, as follows: find $x^{k} \in H$ such that

$$
\left(M^{k} x^{k}, v\right)=-\left(g\left(u^{k}\right), v\right) \text { for all } v \in H,
$$

where $M^{k}$ is a linear mapping from $H$ to $H$, approximating, in some sense, the derivative $g^{\prime}\left(u^{k}\right)$. Then we set

$$
u^{k+1}=u^{k}+t^{k} x^{k}
$$

where $t^{k} \in(0,1]$ is a scalar damping parameter. Setting $M^{k}=g^{\prime}\left(u^{k}\right)$ and $t^{k}=1$ corresponds to Newton's method. 
Generally, a procedure such as (2.4)-(2.5) is intractable computationally since $H$ may be infinite dimensional. Thus we seek to discretize (2.3)-(2.5). Let $\left\{\mathscr{N}_{j}\right\}$ be an indexed family of finite-dimensional subspaces dense in $H$, nested in the sense that $\mathfrak{R}_{j} \subseteq \mathfrak{N}_{k}$ for $k>j$. Let $N_{j}$ denote the dimension of $\mathfrak{M}_{j}$. We assume the dimensions of the spaces increase geometrically,

$$
N_{j}=\beta N_{j-1}, \quad \beta>1,
$$

since this will be the typical situation arising in practice. The discrete analogue of (2.3) is: find $u_{j}^{*} \in \mathfrak{R}_{j}$ such that

$$
\left(g\left(u_{j}^{*}\right), v\right)=0 \quad \text { for all } v \in \Re_{j} .
$$

Once a basis for $\Re_{j}$ has been chosen, (2.7) can be formulated as a set of $N_{j}$ nonlinear algebraic equations.

The analogue of (2.4)-(2.5) proceeds from an initial guess $u_{j}^{0} \in \Re_{j}$ and computes $u_{j}^{k} \in \Re_{j}$ such that

$$
\left(M_{j}^{k} x_{j}^{k}, v\right)=-\left(g\left(u_{j}^{k}\right), v\right) \quad \text { for all } v \in \Re_{j} .
$$

Equation (2.8) corresponds to an $N_{j} \times N_{j}$ linear algebraic system to be solved. Then set

$$
u_{j}^{k+1}=u_{j}^{k}+t_{j}^{k} x_{j}^{k} .
$$

Corresponding to $\Re_{j}$, we define a sequence of seminorms, $|\cdot|_{j}$ on $H$ by

$$
|u|_{j}=\sup _{v \in \pi_{j} ; v \neq 0}|(u, v)| /\|v\| .
$$

In essence, if $u \in H$ and $P_{j}$ is the orthogonal projector from $H$ to $\mathfrak{\Re}_{j}$, then $|u|_{j}=\left\|P_{j}(u)\right\|$; furthermore, since the $\Re_{j}$ are dense in $H$,

$$
\|u\|=\sup _{j}|u|_{j} .
$$

Thus, $|\cdot|_{j}$ represents a strong norm on $\mathfrak{T}_{k}, k \leqslant j$, and $|u|_{j}=\|u\|$ for all $u \in \mathfrak{R}_{k}$, $k \leqslant j$, while $|\cdot|_{j}$ is a seminorm on $\Re_{k}$ with $k>j$. In the solution of (2.7), it is the seminorm $|:|_{j}$ which is computable, and the solution $u_{j}^{*}$ satisfies $\left|g\left(u_{j}^{*}\right)\right|_{j}=0$, while $\left\|g\left(u_{j}^{*}\right)\right\|>0$ in general.

Suppose solutions $u^{*}$ and $u_{j}^{*}$ of (2.3) and (2.7), respectively, exist (this follows from our assumptions below; see Remark 4). Our central assumption is that the discrete solutions $u_{j}^{*}$ are increasingly good approximations of $u^{*}$. Specifically, we assume there exists a fixed constant $C_{1}=C_{1}\left(u, g,\left\{\mathscr{N}_{j}\right\}\right)$ and a positive number $q$ such that

$$
\left\|u^{*}-u_{j}^{*}\right\| \leqslant C_{1} N_{j}^{-q} .
$$

Given (2.12), our strategy for computing approximate solutions which satisfy bounds like (2.12) is to sequentially compute approximate solutions of (2.7), using (2.8)-(2.9), and using the final iterate of the $j-1$ st problem as the initial guess for the $j$ th. We summarize this procedure in

\section{Algorithm I.}

(i) For $j=1$, carry out $s_{1}$ iterations of (2.8)-(2.9), starting from initial guess $u_{1}^{0} \in \mathfrak{K}_{1}$. 
(ii) For $j>1$, carry out $s_{j}$ iterations of (2.8)-(2.9), starting from initial guess $u_{j}^{0}=u_{j-1}^{s_{j-1}} \in \Re_{j-1} \subseteq \Re_{j}$.

3. Analysis. We begin by stating the underlying assumptions of our analysis. Our presentation is chosen to be consistent with our analysis in [5].

Given $u_{1}^{0}$, let $\delta_{j}$ be closed subsets of $\Re_{j}$ inductively defined as follows:

$$
\begin{aligned}
& \delta_{1}=\left\{\left.u \in \mathfrak{K}_{1}|| g(u)\right|_{1} \leqslant\left|g\left(u_{1}^{0}\right)\right|_{1}\right\}, \\
& \delta_{j}=\left\{\left.u \in \mathfrak{R}_{j}|| g(u)\right|_{j} \leqslant \sup _{v \in \delta_{j-1}}|g(v)|_{j}\right\} .
\end{aligned}
$$

Define

$$
S_{0}=\left\{u \in H \mid\|g(u)\| \leqslant \sup _{v \in \delta_{j} ; j \geqslant 1}\|g(v)\|\right\} .
$$

A1. $S_{0}$ is bounded.

Remark 1. For $w \in \mathfrak{M}_{j}, z \in \mathfrak{M}_{j-1}$, and $v \in H$,

$$
|(g(v), w)| \leqslant\left|\left(g(v), P_{j-1} w\right)\right|+\left|\left(g(v)-z,\left(I-P_{j-1}\right) w\right)\right| \text {. }
$$

Hence

$$
|g(v)|_{j} \leqslant|g(v)|_{j-1}+\inf _{z \in \mathscr{N}_{j-1}}|g(v)-z|_{j}
$$

Typically, the spaces $\mathfrak{K}_{j}$ will be such that the second term can be bounded by $C N_{j-1}^{-q}$. Thus if

$$
\gamma_{1}=\left|g\left(u_{1}^{0}\right)\right|_{1}, \quad \gamma_{j}=\sup _{v \in \delta_{j}}|g(v)|_{j}
$$

then

$$
\gamma_{j} \leqslant \gamma_{j-1}+C N_{j-1}^{-q}, \quad j>1
$$

If (2.6) holds,

$$
\gamma_{j} \leqslant \gamma_{1}+C N_{1}^{-q}\left(1-\beta^{-q}\right)^{-1} \leqslant C^{\prime} .
$$

Using (2.11), we see that $S_{0}$ is contained in the level set

$$
S_{0}^{\prime}=\left\{u \in H \mid\|g(u)\| \leqslant C^{\prime}\right\}
$$

(cf. Al of [5]).

A2. We assume $g$ is differentiable on $S_{0}$, and for $u \in S_{0}$ and $v, w \in H$ :

$$
\begin{gathered}
\left|\left(g^{\prime}(u) v, w\right)\right| \leqslant C_{2}\|v\|\|w\|, \\
\inf _{\|v\|=1} \sup _{\|w\| \leqslant 1}\left|\left(g^{\prime}(u) v, w\right)\right| \geqslant k_{3}^{-1}>0, \\
\sup _{v}\left|\left(g^{\prime}(u) v, w\right)\right|>0, \quad w \neq 0
\end{gathered}
$$

( $C_{2}$ is finite and $C_{2}$ and $k_{3}$ are independent of $u$ ).

Remark 2. Equations (3.4)-(3.6) guarantee that a unique solution $v \in H$ will exist for the problem

$$
\left(g^{\prime}(u) v, w\right)=(z, w) \text { for all } w \in H
$$


where $z \in H$ and

$$
\|v\| \leqslant k_{3}\|z\|
$$

see Babuška and Aziz [1, Section 5.2].

A3. For $u \in \mathcal{S}_{j}, v, w \in \Re_{j}$, and $M_{j}^{k}$ as in (2.8), assume

$$
\begin{gathered}
\inf _{\|v\|=1} \sup _{\|w\| \leqslant 1}\left|\left(g^{\prime}(u) v, w\right)\right| \geqslant k_{6}^{-1}>0, \\
\inf _{\|v\|=1} \sup _{\|w\| \leqslant 1}\left|\left(M_{j}^{k} v, w\right)\right| \geqslant k_{1}^{-1}>0
\end{gathered}
$$

( $k_{1}$ and $k_{6}$ are independent of $u$ and $j$ ).

Remark 3. In our particular application (3.8) will follow from A2, and we will show $k_{1} \leqslant 2 k_{6}$ (see inequality 4.7 ).

We embed $S_{0}$ in the closed, convex ball

$$
S_{1}=\left\{u \in H \mid\|u\| \leqslant \sup _{v \in S_{0}}\|v\|+k_{1}\|g(v)\|\right\} .
$$

A4. We assume $g^{\prime}$ is Lipshitz on $S_{1}$ and for $u, v \in S_{1}$,

$$
\left\|g^{\prime}(u)-g^{\prime}(v)\right\| \leqslant k_{2}\|u-v\| \text {. }
$$

Since $g$ is differentiable, we also have

$$
\|g(u)-g(v)\| \leqslant k_{5}\|u-v\|
$$

for $u, v \in S_{1}$ (as in [5, Eq. (2.28)]).

Remark 4. Assumption Al above is analogous to Al in [5]. Equation (3.9) implies a bound as in (3.7), which, in turn, implies A2 of [5]. Finally, A4 above implies A3 of [5]. Thus the argument used to obtain Theorem 1 of [5] implies the existence of each $u_{j}^{*} \in \Re_{j}$ and also $u^{*} \in H$.

We define the relative residuals $\alpha_{j}^{k}$ for the solutions of (2.7) by

$$
\alpha_{j}^{k}=\left|g^{\prime}\left(u_{j}^{k}\right) x_{j}^{k}+g\left(u_{j}^{k}\right)\right|_{j} /\left|g\left(u_{j}^{k}\right)\right|_{j} .
$$

Th quantity $\alpha_{j}^{k}$ is computable and measures how well $x_{j}^{k}$ approximates the true Newton step ( $\alpha_{j}^{k}=0$ for Newton's method). We will choose the damping parameters $t_{j}^{k}$ of (2.9) according to the formula

$$
t_{j}^{k}=\left(1+\mathscr{K}_{j}^{k}\left|g\left(u_{j}^{k}\right)\right|_{j}\right)^{-1}
$$

where the $\mathcal{K}_{j}^{k}$ are nonnegative scalars.

The following result applies Proposition 1 of [5] for each $j \geqslant 1$.

Proposition 3.1. Let $\delta_{0} \in\left(0,1-\alpha_{0}\right), \alpha_{j}^{0} \in\left(0, \alpha_{0}\right), \alpha_{0}<1$, and let $t_{j}^{k}$ be chosen as in (3.14), where $0 \leqslant \mathcal{K}_{j}^{k} \leqslant \mathcal{K}_{0}$, and

$$
\mathscr{K}_{j}^{k} \geqslant\left(k_{1}^{2} k_{2} / 2\right)\left(1-\alpha_{j}^{k}-\delta_{0}\right)^{-1}-\left|g\left(u_{j}^{k}\right)\right|_{j}^{-1} .
$$

Assume A1-A4 and all $\alpha_{j}^{k} \leqslant \alpha_{j}^{0}$. Then

(i) all $u_{j}^{k} \in \mathcal{S}_{j}$, the sequence $\left|g\left(u_{j}^{k}\right)\right|_{j}$ is strictly decreasing, and $\left|g\left(u_{j}^{k}\right)\right|_{j} \rightarrow 0$. Furthermore,

(ii) $\left|g\left(u_{j}^{k+1}\right)\right|_{j} /\left|g\left(u_{j}^{k}\right)\right|_{j} \rightarrow 0$ if and only if $\alpha_{j}^{k} \rightarrow 0$, and, for any fixed $p \in(0,1]$,

$$
\left|g\left(u_{j}^{k+1}\right)\right|_{j} \leqslant C_{3}\left|g\left(u_{j}^{k}\right)\right|_{j}^{1+p}
$$


if and only if

$$
\alpha_{j}^{k} \leqslant C_{4}\left|g\left(u_{j}^{k}\right)\right|_{j}^{p}
$$

for positive constants $C_{3}$ and $C_{4}$.

Note that we may consider $\mathcal{K}_{0}$ as bounded uniformly in $j$ by

$$
\mathscr{K}_{0} \geqslant\left(k_{1}^{2} k_{2} / 2\right)\left(1-\alpha_{0}-\delta_{0}\right)^{-1} \text {. }
$$

Proposition 3.1 states that the approximate-Newton method converges and that the rate of convergence is governed by the $\alpha_{j}^{k}$. The parameter $\delta_{0}$ is a sufficient decrease parameter [5] and can be used in the actual computation to determine if (3.15) is satisfied. In [5] we prove that, for $u_{j}^{k}$ sufficiently close to $u_{j}^{*}$, we have

$$
k_{4}\left\|u_{j}^{k}-u_{j}^{*}\right\| \leqslant\left|g\left(u_{j}^{k}\right)\right|_{j} \leqslant k_{5}\left\|u_{j}^{k}-u_{j}^{*}\right\|,
$$

showing that the rate of convergence of $\left|g\left(u_{j}^{k}\right)\right|_{j}$ to zero is also the asymptotic rate of convergence of $u_{j}^{k}$ to $u_{j}^{*}$.

In our case, however, we are interested in computing $u_{j}^{k}$ only insofar as it is an approximation of $u^{*}$ of (2.3), and not as an approximation of $u_{j}^{*}$ (although the two are clearly related). Thus we want to avoid wasting iterations by computing 'too good' an approximation of $u_{j}^{*}$. In Theorem 3.2, we indicate the degree to which we must approximate $u_{j}^{*}$ in order to obtain bounds of the form (2.12) for the computed solutions.

THEOREM 3.2. Let $u_{j}^{*}$ satisfy (2.7) and let $u_{j}^{k}, 0 \leqslant k \leqslant s_{j}$, be computed as in Algorithm I, using (2.8), (2.9), and (3.14). Let $\delta \in\left(0, \beta^{-q}\right)$, and suppose

$$
\left\|u_{1}^{s_{1}}-u_{1}^{*}\right\| \leqslant C_{1} \varepsilon N_{1}^{-q}
$$

where

$$
\begin{aligned}
& \varepsilon=\delta\left(1+\beta^{q}\right)\left(1-\delta \beta^{q}\right)^{-1}, \\
& \left\|u_{j}^{s_{j}}-u_{j}^{*}\right\| \leqslant \delta\left\|u_{j}^{0}-u_{j}^{*}\right\|,
\end{aligned}
$$

and $u_{j}^{0}=u_{j-1}^{s_{j-1}}, j>1$. Then

$$
\left\|u_{j}^{s_{j}}-u^{*}\right\| \leqslant C_{1}(1+\varepsilon) N_{j}^{-q} .
$$

Proof. Let $e_{j}=\left\|u_{j}^{s_{j}}-u_{j}^{*}\right\|$. Then by (3.18), (2.12), and (2.6),

$$
\begin{aligned}
e_{j} & \leqslant \delta\left\|u_{j}^{0}-u_{j}^{*}\right\| \leqslant \delta\left\{\left\|u_{j-1}^{s_{j-1}}-u_{j-1}^{*}\right\|+\left\|u_{j-1}^{*}-u^{*}\right\|+\left\|u^{*}-u_{j}^{*}\right\|\right\} \\
& \leqslant \delta\left\{e_{j-1}+C_{1}\left(1+\beta^{q}\right) N_{j}^{-q}\right\} .
\end{aligned}
$$

Solution of the majorizing difference equation, and the use of (3.17), shows $e_{j} \leqslant$ $C_{1} \varepsilon N_{j}^{-q}$, and thus

$$
\left\|u_{j}^{s_{j}}-u^{*}\right\| \leqslant e_{j}+\left\|u_{j}^{*}-u^{*}\right\| \leqslant C_{1}(1+\varepsilon) N_{j}^{-q} .
$$

Theorem 3.2 quantifies the advantage of using the strategy embodied in Algorithm I. For each problem after the first, one must reduce the error by only a fixed amount, independent of $j$, in order to obtain a sequence of approximations at the level of discretization error. The central result of this section is that for $j$ sufficiently 
large $s_{j}=1$. Thus, the asymptotic cost of solving the nonlinear systems (2.7) is essentially the cost of computing approximate solutions of linear systems of the form (2.8).

To see this we use a Taylor expansion as in (2.26) of [5] to obtain, for $v \in \mathfrak{M}_{j}$,

$$
\begin{aligned}
0= & \left(g\left(u_{j}^{*}\right), v\right)=\left(g\left(u_{j}^{k}\right), v\right)+\left(g^{\prime}\left(u_{j}^{k}\right)\left\{u_{j}^{*}-u_{j}^{k}\right\}, v\right) \\
& +\int_{0}^{1}\left(\left\{g^{\prime}\left(u_{j}^{k}+s\left\{u_{j}^{*}-u_{j}^{k}\right\}\right)-g^{\prime}\left(u_{j}^{k}\right)\right\}\left\{u_{j}^{*}-u_{j}^{k}\right\}, v\right) d s \\
= & \left(1-t_{j}^{k}\right)\left(g\left(u_{j}^{k}\right), v\right)+t_{j}^{k}\left(g^{\prime}\left(u_{j}^{k}\right) x_{j}^{k}+g\left(u_{j}^{k}\right), v\right) \\
& +\left(g^{\prime}\left(u_{j}^{k}\right)\left\{u_{j}^{*}-u_{j}^{k+1}\right\}, v\right) \\
& +\int_{0}^{1}\left(\left\{g^{\prime}\left(u_{j}^{k}+s\left\{u_{j}^{*}-u_{j}^{k}\right\}\right)-g^{\prime}\left(u_{j}^{k}\right)\right\}\left\{u_{j}^{*}-u_{j}^{k}\right\}, v\right) d s .
\end{aligned}
$$

Moving the third term to the left-hand side, taking (semi) norms, and using (3.8), (3.11), and (3.13), we have

$$
\begin{aligned}
& \left|u_{j}^{k+1}-u_{j}^{*}\right|_{j} \\
& \quad \leqslant k_{6}\left\{\left(1-t_{j}^{k}\right)\left|g\left(u_{j}^{k}\right)\right|_{j}+t_{j}^{k} \alpha_{j}^{k}\left|g\left(u_{j}^{k}\right)\right|_{j}+\left(k_{2} / 2\right)\left|u_{j}^{k}-u_{j}^{*}\right|_{j}^{2}\right\} .
\end{aligned}
$$

Using Proposition 3.1 and (3.15), (3.16), and

$$
\left|g\left(u_{j}^{k}\right)\right|_{j} \leqslant k_{5}\left|u_{j}^{k}-u_{j}^{*}\right|_{j}
$$

(an easy consequence of (3.12), noting that $|v|_{j} \leqslant\|v\|$ with equality for $v \in \mathfrak{R}_{j}$ ), we obtain

$$
\left|u_{j}^{k+1}-u_{j}^{*}\right|_{j} \leqslant k_{6}\left\{\left(\mathscr{K}_{0} k_{5}^{2}+k_{2} / 2\right)\left|u_{j}^{k}-u_{j}^{*}\right|_{j}+k_{5} \alpha_{j}^{k}\right\}\left|u_{j}^{k}-u_{j}^{*}\right|_{j} .
$$

Consider the case $k=0$. Then, using Theorem 3.2 inductively,

$$
\left|u_{j}^{0}-u_{j}^{*}\right|_{j} \leqslant\left\|u_{j-1}^{s_{j-1}}-u^{*}\right\|+\left\|u^{*}-u_{j}^{*}\right\| \leqslant C_{1}\left\{1+(1+\varepsilon) \beta^{q}\right\} N_{j}^{-q},
$$

and, from (3.22),

$$
\left|u_{j}^{1}-u_{j}^{*}\right|_{j} \leqslant\left(C_{6} N_{j}^{-q}+C_{7} \alpha_{j}^{k}\right)\left|u_{j}^{0}-u_{j}^{*}\right|_{j}
$$

where

$$
C_{6}=C_{1} k_{6}\left(\mathscr{K}_{0} k_{5}^{2}+k_{2} / 2\right)\left\{1+(1+\varepsilon) \beta^{q}\right\}, \quad C_{7}=k_{6} k_{5} .
$$

For example, suppose that $j$ is sufficiently large that $C_{6} N_{j}^{-q}<\delta / 2$. Since we can control $\alpha_{j}^{0}$, we may require

$$
C_{7} \alpha_{j}^{0}<\delta / 2
$$

Then (3.18) will be satisfied for $s_{j}=1$. Note that $C_{6}$ and $C_{7}$ are independent of $j$, and thus we have shown

THEOREM 3.3. Let the hypotheses of Proposition 3.1 hold, and suppose $\alpha_{j}^{0}$ is sufficiently small ( $\alpha_{j}^{0}$ satisfies (3.24), for example). Then, for $j$ sufficiently large, we may take $s_{j}=1$ in (3.19). 
We will establish (3.24) for the multilevel iterative method in the next section.

Remark 5. In Algorithm I, we obtain linear convergence of $u_{j}^{s_{j}}$ to $u^{*}$ with the rate of convergence being roughly $\beta^{-q}$. Since Newton's method is quadratically convergent, one can ask under what circumstances we can have $u_{j}^{s_{j}}$ converge to $u^{*}$ quadratically. Assuming (2.12) is sharp, this can be accomplished if we allow the dimensions of the spaces $\mathfrak{K}_{j}$ to square rather than increase geometrically, i.e.,

$$
N_{j}=\beta N_{j-1}^{2}, \quad \beta>0,
$$

rather than (2.6). If we repeat our analysis using (3.25) in place of (2.6), the analogue of Theorem 3.2, Eq. (3.18) would indicate that we must reduce the initial error by $\delta N_{j}^{-q / 2}$ rather than by a fixed amount. If we require $\alpha_{j}^{k} \leqslant C\left|g\left(u_{j}^{k}\right)\right|_{j}$ (which is consistent with quadratic convergence on the basis of Proposition 3.1), then (3.22) implies that the first iteration produces an error reduction of the right order of magnitude $O\left(N_{j}^{-q / 2}\right)$, but the constant may be too large. Two iterations, however, will be more than sufficient; hence $s_{j} \leqslant 2$ for $j$ sufficiently large.

4. A Newton-Multilevel Method. We now return to the example problem (1.1). Let $a \in C^{1}(\bar{\Omega})$ be positive and bounded in $\bar{\Omega}$; i.e.,

$$
0<\underline{\alpha} \leqslant a(x) \leqslant \bar{\alpha} \quad \text { for } x \in \bar{\Omega} .
$$

Let $\partial f / \partial u \in C^{0}(\bar{\Omega})$, and $\partial f / \partial u_{x_{i}} \in C^{1}(\bar{\Omega}), i=1,2$. For $u \in H^{1}(\Omega)$, define

$$
b(u ; v, w)=\int_{\Omega} a \nabla v \cdot \nabla w+b \cdot \nabla v w+c v w d x,
$$

where

$$
b_{i}=\frac{\partial f}{\partial u_{x_{i}}}(x, u, \nabla u) \text { and } c=\frac{\partial f}{\partial u}(x, u, \nabla u)
$$

If we make a correspondence between $a(u, v)$ and $(g(u), v)$ as in Section 2, then $b(u ; v, w)$ corresponds to $\left(g^{\prime}(u) v, w\right)$. Recall that $H=H^{1}(\Omega)$ and that the norm and inner product for $H$ are given in (2.2).

Let $\tau_{1}$ be a quasi-uniform, shape regular triangulation of $\Omega$, and let $h_{1}$ denote the diameter of the largest triangle in $\tau_{1}$ (for convenience, assume $\Omega$ is a polygon). We inductively construct a nested sequence of triangulations $\tau_{j}, j=1,2, \ldots$, as follows: for each triangle $t \in \tau_{j-1}$, construct four triangles in $\tau_{j}$ by pairwise connecting the midpoints of the edges of $t$. Each triangulation will then be quasi-uniform and shape regular, and will have $h_{j}=h_{1} 2^{1-j}$; see [3], [2]. Let $\mathfrak{N}_{j}$ denote the space of $C^{0}$ piecewise linear polynomials associated with $\tau_{j}$. Then $\mathfrak{K}_{j} \subseteq \mathfrak{M}_{k}, k>j$, and $\beta \cong 4$ in (2.6).

The central issue to be addressed in this section is the method of solving the linear systems (2.8) required by Algorithm I. If we were to use Newton's method $\left(M_{j}^{k}=\right.$ $\left.g^{\prime}\left(u_{j}^{k}\right)\right)$, then, in the present context, we would solve the problems: find $\bar{x}_{j}^{k} \in \mathfrak{K}_{j}$ such that

$$
b\left(u_{j}^{k} ; \bar{x}_{j}^{k}, v\right)=-a\left(u_{j}^{k}, v\right) \text { for all } v \in \mathfrak{T}_{j} .
$$

(In this case $\alpha_{j}^{k}=0$ in (3.13).) 
However, rather than solve (4.2) exactly, we will compute an approximate solution, $x_{j}^{k}$, using a multilevel iterative method, in particular, one of the $j$-level schemes described in [3], [2]. In this case, $M_{j}^{k} \neq g^{\prime}\left(u_{j}^{k}\right)$ in general, but rather $M_{j}^{k}$ is defined implicitly in terms of the iteration; see [5, Section 4].

If $r$ iterations of the $j$-level iteration are used, starting from initial guess zero, then the analysis in [3], [2] shows that under suitable hypotheses

$$
\left\|x_{j}^{k}-\bar{x}_{j}^{k}\right\| \leqslant \gamma^{r}\left\|\bar{x}_{j}^{k}\right\|
$$

where $\gamma \in[0,1)$ is a fixed constant independent of $j$. Furthermore, the cost of each iteration is $O\left(N_{j}\right)$ as $j \rightarrow \infty$.

We assume that for $u \in S_{0}$, the boundary value problem: find $v \in H^{1}(\Omega)$ such that

$$
b(u ; v, w)=(z, w) \text { for all } w \in H^{1}(\Omega),
$$

and its adjoint: find $v \in H^{1}(\Omega)$ such that

$$
b^{*}(u ; v, w)=b(u ; w, v)=(z, w) \text { for all } w \in H^{1}(\Omega),
$$

have unique solutions for each $z \in H^{1}(\Omega)$. (This will follow if assumption A2 is satisfied.)

If one assumes (4.4)-(4.5) and a modest amount of elliptic regularity, then one can use the argument in Schatz [14] to prove that the problem: find $v \in \Re_{j}$ such that

$$
b(u ; v, w)=(z, w) \text { for all } w \in \Re_{j},
$$

and its adjoint have unique solutions, provided $h_{1}$ is sufficiently small.

This in turn can be used to verify assumption A3, Eq. (3.8) as follows [1]: Let $v \in H^{1}(\Omega)$ and choose the scalar $\lambda$ sufficiently large that

$$
b(u ; v, v)+\lambda(v, v) \geqslant C\|v\|^{2} .
$$

Note that $\lambda$ is independent of $v$. By arguments given in [14], the problem: find $z \in \Re_{j}$ such that

$$
b(u ; z, w)=(\lambda v, w) \text { for all } w \in \Re_{j},
$$

has a unique solution satisfying $\|z\| \leqslant C^{\prime}\|\lambda v\|$, provided $h_{1}$ is sufficiently small.

Now let $v \in \Re_{j}$ with $\|v\|=1$, and let $z$ be defined as above. Take

$$
w=(v+z) /\left(1+C^{\prime} \lambda\right) \text {, }
$$

and note that $\|w\|>1$. Then

$$
\begin{aligned}
b(u ; v, w) & =(b(u ; v, v)+b(u ; v, z)) /\left(1+C^{\prime} \lambda\right) \\
& =(b(u ; v, v)+\lambda(v, v)) /\left(1+C^{\prime} \lambda\right) \\
& \geqslant C /\left(1+C^{\prime} \lambda\right) \equiv k_{6}^{-1} .
\end{aligned}
$$

Finally, note that, on the basis of (4.3),

$$
\begin{aligned}
\left|\left(M_{j}^{k}\right)^{-1}\right|_{j} & \leqslant\left|g^{\prime}\left(u_{j}^{k}\right)^{-1}\right|_{j}+\left|\left(M_{j}^{k}\right)^{-1}-g^{\prime}\left(u_{j}^{k}\right)^{-1}\right|_{j} \\
& \leqslant\left(1+\gamma^{r}\right)\left|g^{\prime}\left(u_{j}^{k}\right)^{-1}\right|_{j} \leqslant\left(1+\gamma^{r}\right) k_{6},
\end{aligned}
$$

showing that we may take $k_{1}=2 k_{6}$ in $\mathrm{A} 3$, Eq. (3.9). 
We want to choose $r$ such that the hypotheses of Theorem 3.3 will be satisfied and we can take $s_{j}=1$ for large enough $j$. Observe that

$$
\begin{aligned}
\left|g^{\prime}\left(u_{j}^{k}\right) x_{j}^{k}+g\left(u_{j}^{k}\right)\right|_{j} & =\sup _{v \in \mathscr{R}_{j}}\left|b\left(u_{j}^{k} ; x_{j}^{k}, v\right)+a\left(u_{j}^{k}, v\right)\right| /\|v\| \\
& =\sup _{v \in \mathscr{R}_{j}}\left|b\left(u_{j}^{k} ; x_{j}^{k}-\bar{x}_{j}^{k}, v\right)\right| /\|v\| \\
& \leqslant C_{2}\left\|x_{j}^{k}-\bar{x}_{j}^{k}\right\| \leqslant C_{2} \gamma^{r}\left\|\bar{x}_{j}^{k}\right\| \\
& =C_{2} \gamma^{r}\left|\bar{x}_{j}^{k}\right|_{j} \leqslant C_{2} \gamma^{r} k_{6}\left|g\left(u_{j}^{k}\right)\right|_{j},
\end{aligned}
$$

where we have used (3.4), (3.8), and (4.3). Thus, from (3.13),

$$
\alpha_{j}^{k} \leqslant C_{2} k_{6} \gamma^{r}
$$

To apply Theorem 3.3, we must have $\alpha_{j}^{k}$ sufficiently small that an inequality like (3.24) holds. To insure (3.24), we can require that $r$ be sufficiently large that

$$
C_{7} C_{2} k_{6} \gamma^{r} \leqslant \delta / 2
$$

Note that $r$ can be chosen independent of $j$.

Since $s_{j}=1$ asymptotically, the bulk of the work per level consists of constructing the linear system (4.2), and then carrying out $r$ iterations of the $j$-level scheme. Since both of these are asymptotically $O\left(N_{j}\right)$ processes, the work per level can be bounded by, say, $C_{8} N_{j}$ operations. The cumulative work for levels 1 to $j$ can then be bounded by

$$
\sum_{k \leqslant j} C_{8} N_{k} \leqslant C_{8} N_{j}\left\{1+\beta^{-1}+\beta^{-2}+\cdots\right\} \leqslant C_{8} N_{j}\left(1-\beta^{-1}\right)^{-1},
$$

due to (2.6). We summarize in

THEOREM 4.1. Let Algorithm I be implemented using the j-level iteration, and assume that (4.3) and the hypotheses of Theorem 3.3 hold. Then, for $j$ sufficiently large and $h_{1}$ sufficiently small,

$$
\left\|u_{j}^{1}-u^{*}\right\| \leqslant C_{1}(1+\varepsilon) N_{j}^{-q}
$$

as in Eq. (3.19). Furthermore, the computation of $u_{j}^{1} \in \mathfrak{R}_{j}$, including all previous computations in $\mathfrak{K}_{k}, k \leqslant j-1$, requires $O\left(N_{j}\right)$ time.

5. A Numerical Illustration. We consider the mildly nonlinear elliptic equation

$$
\begin{aligned}
-\Delta u+u\left(u_{x}+u_{y}\right)+f(x, y) & =0 & & \text { in } \Omega=(0,1) \times(0,1), \\
u & =g & & \text { on } \partial \Omega,
\end{aligned}
$$

where $f$ and $g$ are chosen such that the solution is $u^{*}=e^{-10 x y}$.

This problem was solved using the Fortran program PLTMG [6]. This package implements the scheme described in Section 4. The initial grid was the uniform $5 \times 5$ mesh given in Figure 5.1. 


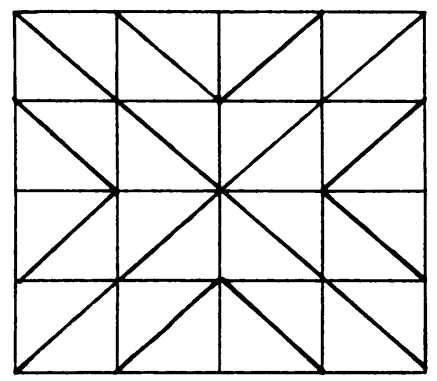

FIGURE 5.1. $\tau_{1}$

Two uniform refinements of $\tau_{1}$ were made, giving $\tau_{2}$ and $\tau_{3}$ as $9 \times 9$ and $17 \times 17$ grids, respectively. The computations were done on a Vax computer. The initial guess for the level -1 problem was $u_{1}^{0}=0$, although better initial guesses are easy to construct. With this initial guess $s_{1}=4$ was sufficient to reduce the error in the discrete level -1 system by about $10^{-6}$, i.e.,

$$
\left\|u_{1}^{4}-u_{1}^{*}\right\| \sim 10^{-6}\left\|u_{1}^{*}\right\|
$$

where $\|\cdot\|$ is the $H^{1}(\Omega)$ norm. Thus, for practical purposes, the level -1 problem was solved exactly.

We solved the problem on the second and third grids using Algorithm I for $s_{j}=1$, $j>1$, and $s_{j}=2, j>1$. The relative error was computed from

$$
\text { correct digits }=-\log \left(\left\|u_{j}^{s_{j}}-u^{*}\right\| /\left\|u^{*}\right\|\right),
$$

where $u^{*}$ is the solution of the continuous problem. The results of the calculation are summarized in Table 5.1. Taking $s_{j}>2$ does not change the results; also $t_{j}^{k}=1$ for all steps.

TABLE 5.1

\begin{tabular}{cccc} 
level & $N$ & \multicolumn{2}{c}{ Correct digits } \\
& & $s_{1}=4, s_{2}=s_{3}=1$ & Case 2 \\
& & .334 & $s_{1}=4, s_{2}=s_{3}=2$ \\
1 & 25 & .576 & .334 \\
2 & 81 & .859 & .576 \\
3 & 289 & .859
\end{tabular}

Since we are comparing the computed solution with the solution of the continuous problem, the measured error includes both the discretization error $u_{j}^{*}-u^{*}$ and error from the solution process $u_{j}^{s_{j}}-u_{j}^{*}$. The identical results for $s_{j}=1$ and $s_{j}=2$ indicate that the measured error is essentially all discretization error. Thus, in this problem, taking $s_{j}=1, j>1$, was sufficient to produce computed solutions at the level of discretization error (although taking $s_{j}>1$ produced better approximations of the discrete solutions $u_{j}^{*}$ ). Although one cannot expect to have $s_{j}=1$ for $j>1$ always, this example shows that the asymptotic behavior predicted by Theorem 3.3 can actually be achieved in problems of practical size. 
The nonlinear package has also been successfully applied to much more complicated problems of physical interest; see, for example, Hutson [11].

Department of Mathematics

University of California at San Diego

La Jolla, California 92093

Bell Telephone Laboratories

Murray Hill, New Jersey 07974

1. Ivo BABUSKa \& A. K. Azız, "Survey lectures on the mathematical foundations of the finite element method," in The Mathematical Foundations of the Finite Element Method with. Applications to Partial Differential Equations, A. K. Aziz, Ed., Academic Press, New York, 1972, pp. 111-184.

2. Randolph E. BANK \& TodD F. Dupont, “An optimal order process for solving finite element equations," Math. Comp., v. 36, 1981, pp. 35-51.

3. RANDOLPH E. BANK, "A comparison of two multi-level iterative methods for non-symmetric and indefinite elliptic finite element equations," SIAM J. Numer. Anal., v. 18, 1981, pp. 724-743.

4. Randolph E. Bank \& Donald J. Rose, "Parameter selection for Newton-like methods applicable to nonlinear partial differential equations," SIAM J. Numer. Anal., v. 17, 1980, 806-822.

5. Randolph E. Bank \& Donald J. Rose, "Global approximate Newton methods," Numer. Math., v. 37, 1981, pp. 279-295.

6. Randolph E. BANK \& ANDREW H. Sherman, "Algorithmic aspects of the multi-level solution of finite element equations," in Sparse Matrix Proceedings-1978, (I. S. Duff and G. W. Stewart, Eds.), SIAM, Philadelphia, Pa., 1979, pp. 62-89.

7. ACHi BrandT, "Multi-level adaptive solutions to boundary value problems," Math. Comp., v. 31, 1977, pp. 333-390.

8. AChi Brandt \& Steve McCormick, Private communication, 1980.

9. Wolfgang HackBusch, On the Convergence of a Multi-Grid Iteration Applied to Finite Element Equations, Technical Report 77-8, Mathematisches Institut, Universität zu Köln, 1977.

10. Wolfgang HaCKBusCh, "On the fast solution of nonlinear elliptic equations," Numer. Math., v. 32, 1979, pp. 83-95.

11. A. R. Hutson, "Role of dislocations in the electrical conductivity of cds," Phys. Rev. Lett., v. 46, 1981, pp. 1159-1162.

12. Lois MANSFIELD, "On the solution of nonlinear finite element systems," SIAM J. Numer. Anal., v. 17, 1980, pp. $752-765$.

13. R. A. Nicolaides, "On the $l^{2}$ convergence of an algorithm for solving finite element systems," Math. Comp., v. 31, 1977, pp. 892-906.

14. Alfred H. Schatz, "An observation concerning Ritz-Galerkin methods with indefinite bilinear forms," Math. Comp., v. 28, 1974, pp. 959-962. 